\title{
Salicylic acid amplifies Carbachol-induced bronchoconstriction in human precision-cut lung slices
}

Joseph Jude ${ }^{1^{*}}$, Danielle Botelho ${ }^{2}$, Nikhil Karmacharya ${ }^{1}$, Gao Yuan Cao ${ }^{1}$, William Jester ${ }^{1}$ and Reynold A. Panettieri $\mathrm{Jr}^{1}$

\begin{abstract}
Background: Asthma exacerbations evoke emergency room visits, progressive loss of lung function and increased mortality. Environmental and industrial toxicants exacerbate asthma, although the underlying mechanisms are unknown. We assessed whether 3 distinct toxicants, salicylic acid (SA), toluene diisocyanate (TDI), and 1-chloro-2,4dinitrobenzene (DNCB) induced airway hyperresponsiveness (AHR) through modulating excitation-contraction coupling in human airway smooth muscle (HASM) cells. The toxicants include a non-sensitizing irritant (SA), respiratory sensitizer (TDI) and dermal sensitizer (DNCB), respectively. We hypothesized that these toxicants induce AHR by modulating excitation-contraction (EC) coupling in airway smooth muscle (ASM) cells.
\end{abstract}

Methods: Carbachol-induced bronchoconstriction was measured in precision-cut human lung slices (hPCLS) following exposure to SA, TDI, DNCB or vehicle. Culture supernatants of hPCLS were screened for mediator release. In HASM cells treated with the toxicants, surrogate readouts of EC coupling were measured by phosphorylated myosin light chain (pMLC) and agonist-induced $\mathrm{Ca}^{2+}$ mobilization $\left(\left[\mathrm{Ca}^{2+}\right]_{\mathrm{i}}\right)$. In addition, Nrf-2-dependent antioxidant response was determined by NAD(P) H quinone oxidoreductase 1 (NQO1) expression in HASM cells.

Results: In hPCLS, SA, but not TDI or DNCB, potentiated carbachol-induced bronchoconstriction. The toxicants had little effect on release of inflammatory mediators, including IL-6, IL-8 and eotaxin from hPCLS. In HASM cells, TDI amplified carbachol-induced MLC phosphorylation. The toxicants also had little effect on agonist-induced $\left[\mathrm{Ca}^{2+}\right]_{\mathrm{i}}$.

Conclusion: SA, a non-sensitizing irritant, amplifies agonist-induced bronchoconstriction in hPCLS via mechanisms independent of inflammation and $\mathrm{Ca}^{2+}$ homeostasis in HASM cells. The sensitizers TDI and DNCB, had little effect on bronchoconstriction or inflammatory mediator release in hPCLS.

Implications: Our findings suggest that non-sensitizing irritant salicylic acid may evoke AHR and exacerbate symptoms in susceptible individuals or in those with underlying lung disease.

\section{Background}

Asthma exacerbations evoke emergency room visits, deterioration in lung function and even mortality. Chemical toxicants can trigger asthma exacerbations. The underlying mechanisms of toxicant-associated exacerbations remain unknown. Inflammation, oxidative injury and immune sensitization are putative mechanisms through which toxicants elicit AHR, although compelling evidence suggest that some toxicants induce AHR uncoupled from

\footnotetext{
* Correspondence: joseph.antonyjude@rutgers.edu

${ }^{1}$ Rutgers Institute for Translational Medicine and Science (RITMS), Rutgers,

The State University of New Jersey, Rm\# 4276, 89 French Street, New

Brunswick NJ08901, USA

Full list of author information is available at the end of the article
}

inflammation [1]. ASM is a pivotal tissue regulating bronchomotor tone and a primary modulator of bronchoconstriction in asthma and COPD.

To assess whether ASM function is differentially modulated by toxicants, we chose to study the effects of a non-sensitizing irritant, a respiratory or a dermal sensitizer on excitation-contraction (EC) coupling in human ASM (HASM) cells. The non-sensitizing irritant salicylic acid (SA) is a plant extract used as an exfoliating agent in cosmetic products. Irritant-induced asthma is a category of occupational asthma characterized by lack of sensitization or adaptive immune response [2-4]. Sensitizers rely on innate and adaptive immune responses with antibody

(C) The Author(s). 2019 Open Access This article is distributed under the terms of the Creative Commons Attribution 4.0 International License (http://creativecommons.org/licenses/by/4.0/), which permits unrestricted use, distribution, and 
production to elicit their adverse reaction. Toluene diisocyanate (TDI), a manufacturing intermediate in many synthetic materials, is a respiratory sensitizer and a commonly reported cause of occupational asthma [5, 6]. Since 1970s, there is a decline in TDI-induced occupational asthma incidence rates, largely due to engineering controls at work places to minimize exposure [7]. However, studies seeking to determine exposure-response relationship found that cumulative exposure to lower levels of TDI can accelerate decline in lung function (measured by forced expiratory volume in $1 \mathrm{~s}-\mathrm{FEV}_{1}, \quad$ [8-10]. Dinitrochlorobenzene (DNCB) and its chemical variants are dermal sensitizers. Innate and adaptive immune cellular responses are implicated in DNCB-induced contact hypersensitivity (reviewed in [11]). In animal models, inhaled DNCB reportedly elicit allergic inflammation and sensitization with little effect on breathing mechanics [12-15].

ASM cells play a pivotal role in AHR through their synthetic, mechanical and remodeling functions $[16,17]$. In ASM cells, agonist-induced mobilization of $\mathrm{Ca}^{2+}$ from intracellular stores elevates cytosolic $\mathrm{Ca}^{2+}\left(\left[\mathrm{Ca}^{2+}\right]_{\mathrm{i}}\right)$, which in turn binds to calmodulin and activates myosin light chain (MLC) kinase [18, 19]. MLC kinase phosphorylates MLC and induces actomyosin cross-bridge cycling and ASM cell shortening (reviewed in [20]). Alternatively, $\mathrm{Ca}^{2+}$ sensitization mechanism inhibits MLC phosphatase activity and sustains elevated p-MLC level to increase actomyosin cross-bridge cycling [21]. Therefore, agonist-induced $\left[\mathrm{Ca}^{2+}\right]_{\mathrm{i}}$ and MLC phosphorylation levels are considered the surrogate measures of EC coupling in ASM cells. Others and we have reported that toxicants can modulate these signaling pathways to increase bronchomotor tone or elicit AHR [1, 22-24]. Recent evidence also suggests that actin polymerization and cytoskeletal reorganization could elicit ASM cell shortening independent of cellular $\mathrm{Ca}^{2+}$ homeostasis and MLC phosphorylation [25].

In this study, using precision-cut human lung slices (hPCLS) and primary human airway smooth muscle (HASM) cells, we tested the central hypothesis that the toxicants SA, TDI and DNCB induce AHR by modulating EC coupling in ASM cells. Our findings show that the non-sensitizing irritant SA induces AHR independent of inflammatory mediator release, while TDI or DNCB has little effect on AHR or inflammatory mediator release. The sensitizer TDI increased agonist-induced MLC phosphorylation in HASM cells, indicating modulation of EC coupling in HASM cells.

\section{Materials and methods Reagents}

HAM's F-12 medium, PBS, FBS, 0.05\% Trypsin and EDTA, PAGE/western blotting supplies and Lipofectamine RNAimax were purchased from Life Technologies
(Grand Island, NY). Antibodies for pMLC ( $\mathrm{p}^{\mathrm{S} 18 /}$ ${ }^{\mathrm{T} 19}$-MLC), total MLC and tubulin were purchased from Cell Signaling Technology (Danvers, MA). Antibody for NQO1 were purchased from Santa Cruz Biotechnology (Santa Cruz, CA). Duoset ELISA kits for IL-6, IL-8 and eotaxin were obtained from R\&D Biosystems (Minneapolis, MN). SiRNA were purchased from Dharmacon (Lafayatte, CO). Fluo-8 calcium flux assay kit was purchased from abcam (Cambridge, MA). LDH cytotoxicity assay kit was purchased from Thermo scientific (Rockford, IL). All other reagents, including the 3 toxicants, were purchased from Sigma Aldrich (St.Loius, MO).

\section{Culture of HASM cells}

Primary HASM cells were harvested, characterized and grown in culture as described in our previous publications [26]. Cells were used in experiments within the first 4 passages to ensure proper smooth muscle phenotype. HASM cells were serum-deprived for $48 \mathrm{~h}$ prior to experimental exposures.

\section{Human precision-cut lung slices (hPCLS) and carbachol concentration-response}

Normal human lungs were obtained through National Disease Research Interchange (NDRI, Philadelphia, PA) or International Institute for the Advancement of Medicine (IIAM, Edison, NJ). Samples were de-identified and therefore exempted by the Rutgers University Institutional Review Board. PCLS were prepared as previously described [27, 28]. Carbachol concentration-response experiments were conducted by exposing hPCLS containing small airways to incremental concentrations of carbachol $\left(10^{-8}-10^{-4} \mathrm{M}\right)$ for $10 \mathrm{~min}$ each and capturing airway luminal narrowing by a supravital microscope. Airway luminal area for each CCh concentration was expressed as the percentage of baseline luminal area. Semi-logarithmic concentration response curves were used to determine agonist concentration of half-maximal response $\left(\log \mathrm{EC}_{50}\right)$, maximal response $\left(\mathrm{E}_{\max }\right)$ and area under the curve (AUC) as the pharmacological parameters.

\section{Exposure to toxicants}

Human PCLS or HASM cells were exposed to toxicants in F-12 culture medium. Salicylic acid (SA) was dissolved in serum-free F-12 medium. TDI and DNCB stock solutions were prepared in 200-proof ethanol. All subsequent dilutions were made in serum-free F-12 medium. The following concentrations of the toxicants were used in hPCLS: SA, 0.1, 1 \& 10 uM; TDI, 0.01, 0.1 \& $1 \mathrm{uM}$; DNCB 0.1, 1 \& $10 \mathrm{uM}$. HPCLS or HASM cells were exposed to toxicants $24 \mathrm{~h}$ before determining agonist-induced bronchoconstriction, mediator release, MLC phosphorylation or $\mathrm{Ca}^{2+}$ mobilization. HASM cells 
were exposed to $10 \mathrm{uM}$ carbachol for $10 \mathrm{~min}$ to determine MLC phosphorylation. Concentrations of the toxicants were determined partly based on published cytotoxicity data [29]. Viability of hPCLS or HASM cells in the presence of toxicants were assessed by LDH activity level in culture supernatants (Fig. 2d \& e). In hPCLS, preliminary experiments were conducted to determine whether each toxicant, in their highest non-toxic concentration, has effect on bronchoconstriction in hPCLS a) upon acute $(10 \mathrm{~min})$ exposure, and b) following $24 \mathrm{~h}$ exposure. Since acute exposure of each toxicant had little effect on bronchoconstriction, prolonged exposure regime was used for the remainder of the study. In HASM cells, the toxicants were used at 10-fold lesser concentration than what is used in hPCLS.

\section{Determination of $\left[\mathrm{Ca}^{2+}\right]_{\mathrm{i}}$ in HASM cells}

Agonist-induced $\left[\mathrm{Ca}^{2+}\right]_{\mathrm{i}}$ in HASM cells was determined as previously described [1] with some modifications. Briefly, HASM cells grown to confluence in a 48-well plate were loaded with fluo-8 $\mathrm{Ca}^{2+}$-binding dye. Carbachol $(10 \mathrm{uM})$ or histamine $(1 \mu \mathrm{M})$ were used to elicit $\mathrm{Ca}^{2+}$ response in HASM cells. Fluorescence intensity was monitored for up to 2 min following agonist injection. Area under the curve (AUC) of the time-dependent fluorescence (relative fluorescence units- RFU) was calculated from the response curve.

\section{Mediator release from PCLS}

Preliminary screening for inflammatory mediators was performed using a custom-designed Luminex ${ }^{\circ}$ multianalyte array to measure 12 cytokines and chemokines listed in Additional file 1: Table S1 (R\&D Systems, Minneapolis, $\mathrm{MN}$ ). The toxicants had little effect on release of those 12 mediators from hPCLS (data not shown). Subsequently, IL-6, IL-8 and eotaxin were measured in hPCLS supernatants using Duo-Set ELISA kits following manufacturer's instructions. TNF- $\alpha(10 \mathrm{ng} / \mathrm{ml})$ and IL-13 $(100 \mathrm{ng} / \mathrm{ml})$ were used as the positive controls for induction of IL-6/IL-8 and Eotaxin, respectively. BCA protein assay was used to determine the protein concentration in the supernatants and the analyte quantity was normalized to the total protein in the supernatant. LDH activity in the supernatants were measured following manufacturer's protocol (Pierce LDH cytotoxicity say kit, Thermo Scientific, Rockford, IL). One \% Triton-X 100 was used as the positive control to induce maximal LDH release. In HASM cells, $100 \mu \mathrm{M}$ ceramide was used as the positive control to induce maximal LDH release.

\section{Transfection of HASM cells}

HASM cells were transiently transfected with $50 \mathrm{nM}$ of non-targeting or Nrf-2-targeting siRNA as previously described [1]. Cells were exposed to DNCB (0.1 and 1 uM) $72 \mathrm{~h}$ post-transfection.

\section{Data analysis}

HASM cells or hPCLS from at least 3 donors were used in the experiments ( $n=3$ donors). In hPCLS studies, from each donor, minimum 3 slices per experimental group were used as technical replicates. Data are expressed as mean or mean \pm SEM. GraphPad Prism 5.0 was used for statistical analysis and means were considered significantly different when $p \leq 0.05$.

\section{Results}

\section{Salicylic acid enhances carbachol-induced} bronchoconstriction in hPCLS

To determine whether exposure to toxicants SA, TDI or DNCB potentiated carbachol-induced bronchoconstriction, hPCLS were exposed to vehicle or toxicants for $24 \mathrm{~h}$ and carbachol-induced airway narrowing was determined. SA (10 uM) significantly decreased the log $\mathrm{EC}_{50}$ (Fig. 1a \& b) of the carbachol concentration response curve, suggesting SA enhanced the sensitivity of the airways to the contractile agonist. SA, however, has little effect on the maximal response $\left(E_{\max }\right)$ or area under the curve (AUC) of the concentration-response curve (Fig. 1c \& d). Neither TDI nor DNCB exposure had any significant effect on carbachol-induced bronchoconstriction (Fig. 1e-h, Additional file 2: Figure S1).

\section{Salicylic acid or toluene diisocyanate has little effect on inflammatory mediator release from hPCLS}

Irritants and sensitizers induce a variety of inflammatory mediators in target tissues to mediate toxicant injury [30]. Since inflammatory mediators are known to induce airway hyper-reactivity [31, 32], we postulated that toxicants SA and TDI may elicit inflammatory mediator release from hPCLS. Lung slices were treated with vehicle, SA or TDI for $24 \mathrm{~h}$ and culture supernatants were initially screened for 12 mediators using a custom-designed Luminex ${ }^{\circ}$ cytokine/chemokine array (Additional file 1: Table S1). SA, TDI or DNCB had little effect on the levels of any of the 12 analytes screened (data not shown). The culture supernatants were further analyzed for their IL-6, IL-8 and eotaxin levels using ELISA. SA (10 uM) or TDI ( $1 \mathrm{uM})$ had little effect on IL-6, IL-8 or eotaxin release from hPCLS (Fig. 2 a-c). As a positive control, pro-inflammatory cytokine TNF- $\alpha(10 \mathrm{ng} / \mathrm{ml})$, significantly increased release of IL-6 and IL-8 from hPCLS, while IL-13 (100 $\mathrm{ng} / \mathrm{ml}$ ) increased the eotaxin levels. LDH activity assay in the culture supernatants showed that neither SA nor TDI decreased viability of hPCLS (Fig. 2d). The toxicants caused $<30 \%$ reduction of viability in HASM cells, when compared to ceramide (Fig. 2e). 
a

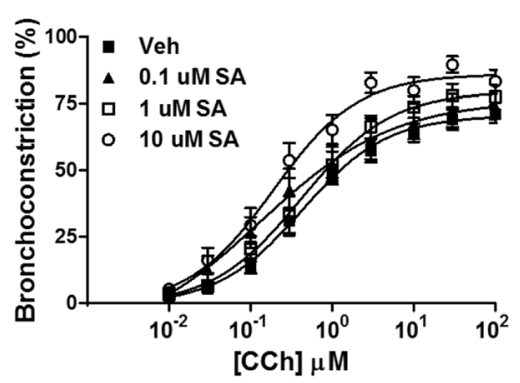

C

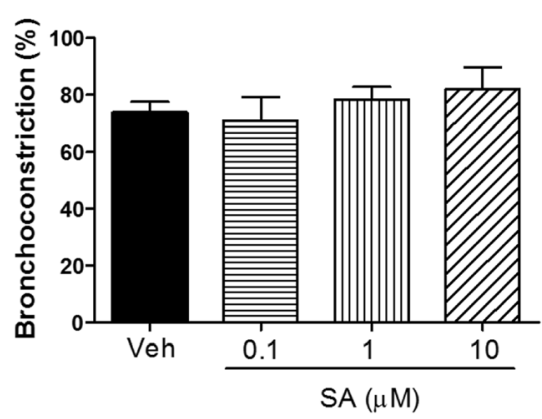

e

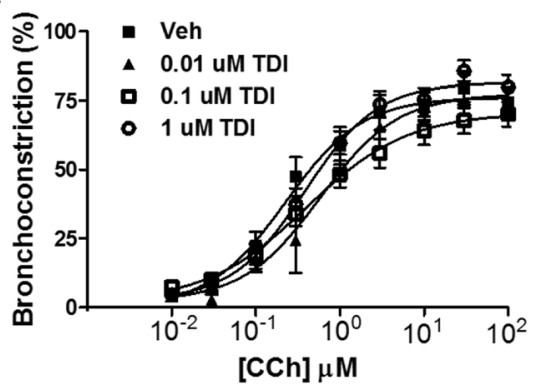

g

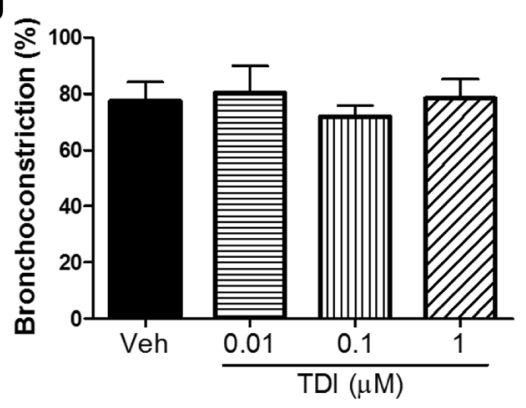

b

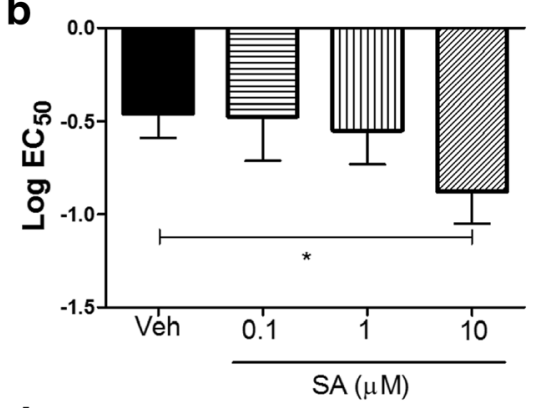

d

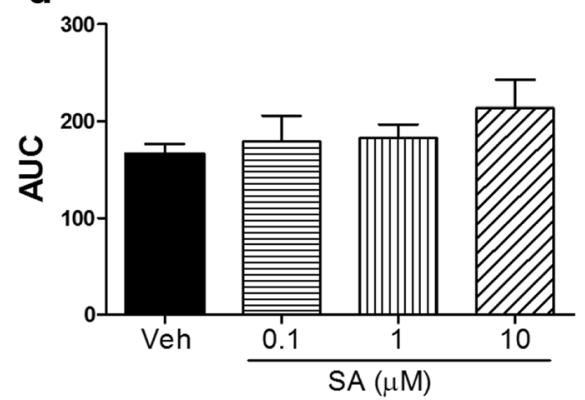

f

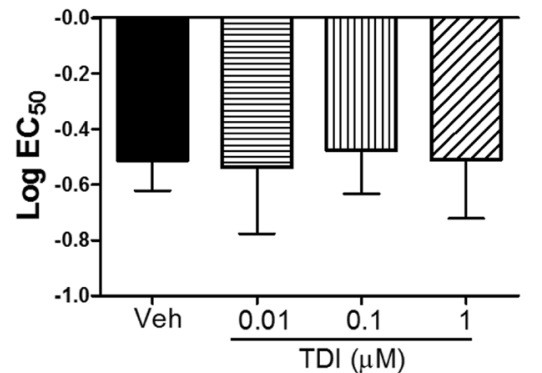

h

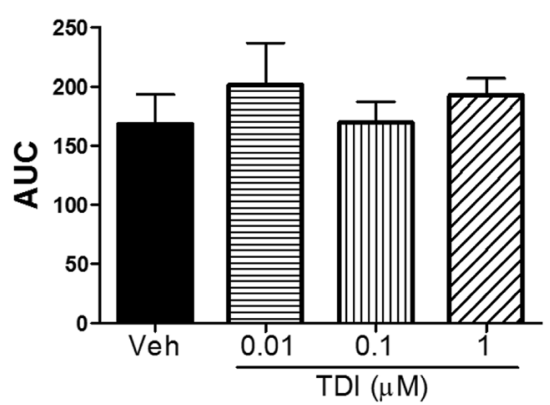

Fig. 1 Salicylic acid enhances carbachol-induced bronchoconstriction in hPCLS. Precision-cut human lung slices (hPCLS) from normal human lung donors were exposed to salicylic acid (SA, vehicle F-12 medium) or toluene diisocyanate (TDI, vehicle $0.1 \%$ ethanol) for $24 \mathrm{~h}$, followed by a carbachol (CCh) concentration-response for bronchoconstriction. $\mathbf{a} \&$ b) SA (10 uM) significantly increased CCh-induced bronchoconstriction $\left(n=4-5\right.$ donors, Log EC $50,{ }^{*} p=$ 0.04 Veh vs SA 10 uM). e-h) TDI has no significant effect on CCh-induced bronchoconstriction ( $n=3-6$ donors, Log EC $50, p=0.4$ Veh Vs TDI 1 uM)

TDI enhances carbachol-induced phosphorylation of myosin light chain in HASM cells

Salicylic acid-induced airway hyperresponsiveness (AHR) may be mediated through its direct effect on EC coupling in HASM cells. Our previous studies reported that the indoor air pollutant formaldehyde modulates EC coupling in HASM cells to elicit AHR [1]. To test this possibility, HASM cells were exposed to SA, TDI or DNCB for $24 \mathrm{~h}$ and myosin light chain (MLC) phosphorylation was determined in the presence or absence of carbachol. TDI (1 uM) significantly attenuated the baseline level of MLC phosphorylation, while enhancing carbachol-induced 

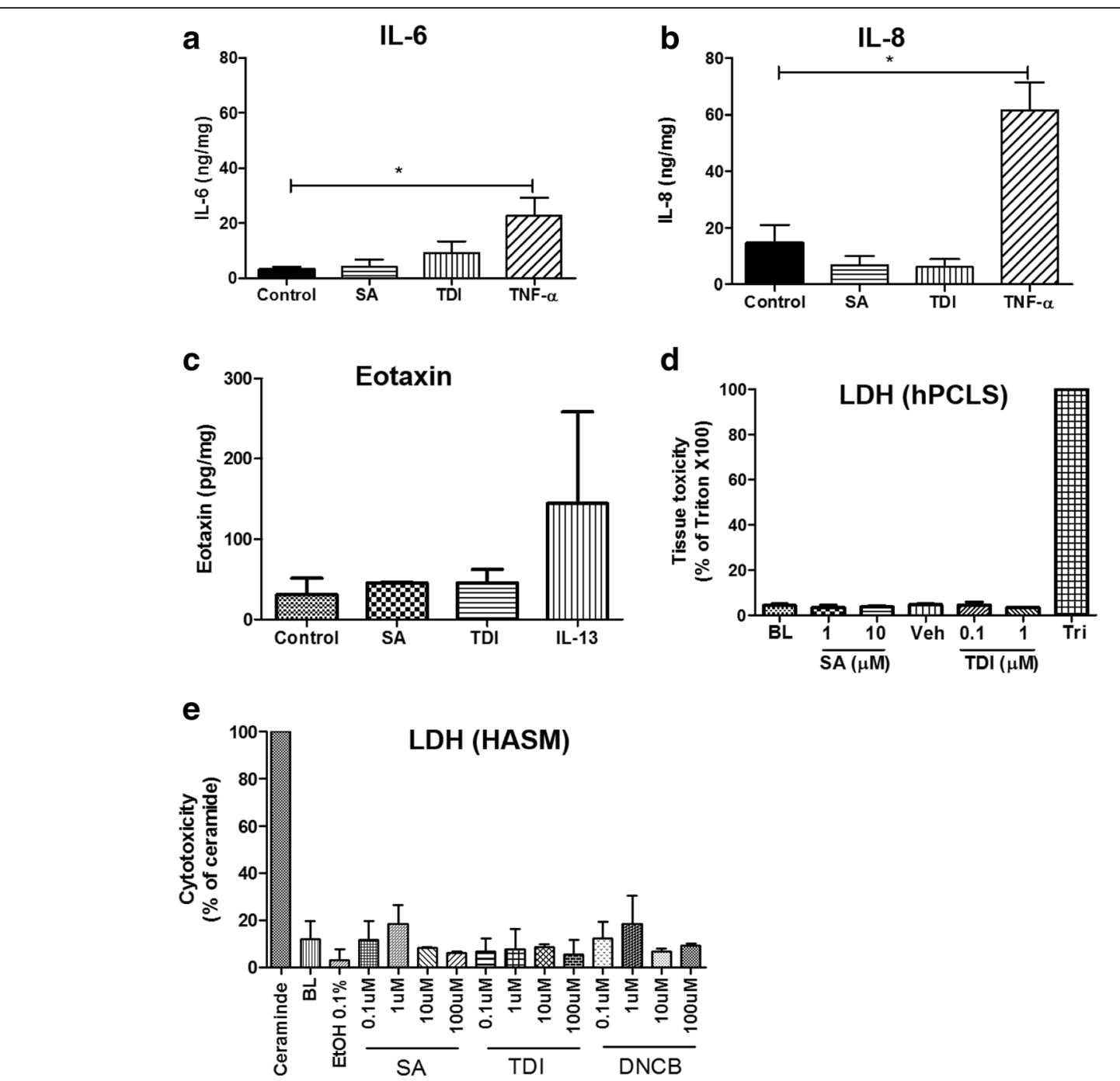

Fig. 2 Salicylic acid or toluene diisocyanate has little effect on inflammatory mediator release from hPCLS. Supernatants from human PCLS exposed to salicylic acid (SA $10 \mathrm{uM}$, vehicle F-12 medium) or toluene diisocyanate (TDI $1 \mathrm{uM}$, vehicle $0.1 \%$ ethanol) for $24 \mathrm{~h}$ were screened using a custom Luminex cytokine/chemokine mediator array (Additional file 1: Table S1) to determine mediator release. SA or TDI had no significant effect on any of the 12 mediators analyzed by the array (data not shown). In additional ELISA assays, SA or TDI had little effect on a) IL-6, b) IL-8 or c) Eotaxin release from hPCLS, while the positive controls TNF-a $(10 \mathrm{ng} / \mathrm{ml})$ or IL-13 (100 ng/ml) increased IL-6, IL-8 and eotaxin levels, respectively. d) SA or TDI has little effect in tissue viability measured by LDH activity in culture supernatant (mean \pm SEM of $n=4-5$ donors; BL-baseline, Tri-1\% Triton X-100). E) The toxicants had $<30 \%$ cytotoxicity at $0.1-100 \mu M(24 \mathrm{~h}$ ) concentration range in HASM cells, measured by LDH activity in culture supernatants (Mean \pm SD of $n=2$ donors; BL-baseline)

MLC phosphorylation (Fig. 3 a-c). SA (Fig. 3 d-f) or DNCB (Fig. $3 \mathrm{~g}$-i) had little effect on baseline or carbachol-induced MLC phosphorylation.

\section{Salicylic acid or toluene diisocyanate has little effect on agonist-induced $\left[\mathrm{Ca}^{2+}\right]_{i}$ in HASM cells}

In HASM cells, agonist-induced elevation of cytosolic $\mathrm{Ca}^{2}$ ${ }^{+}\left(\left[\mathrm{Ca}^{2+}\right]_{\mathrm{i}}\right)$ is a pivotal step in excitation contraction coupling. Amplified $\mathrm{Ca}^{2+}$ mobilization mechanisms are implicated in airway hyperreactivity [33-35]. To determine whether augmented $\left[\mathrm{Ca}^{2+}\right]_{\mathrm{i}}$ mediates SA-induced AHR and TDI effects on MLC phosphorylation, HASM cells treated with these toxicants were stimulated with contractile agonists carbachol or histamine and $\left[\mathrm{Ca}^{2+}\right]_{i}$ was determined. SA or TDI pre-treatment has little effect on carbachol or histamine-induced $\left[\mathrm{Ca}^{2+}\right]_{\mathrm{i}}$ (Fig. 4). DNCB had little effect on carbachol or histamine-induced $\mathrm{Ca}^{2+}$ mobilization (data not shown).

\section{DNCB induces Nrf-2-dependent anti-oxidant response in HASM cells}

Oxidative stress is a common mechanism of injury by many respiratory toxicants. Since, DNCB has little effect on any of the experimental readouts in HASM cells and 


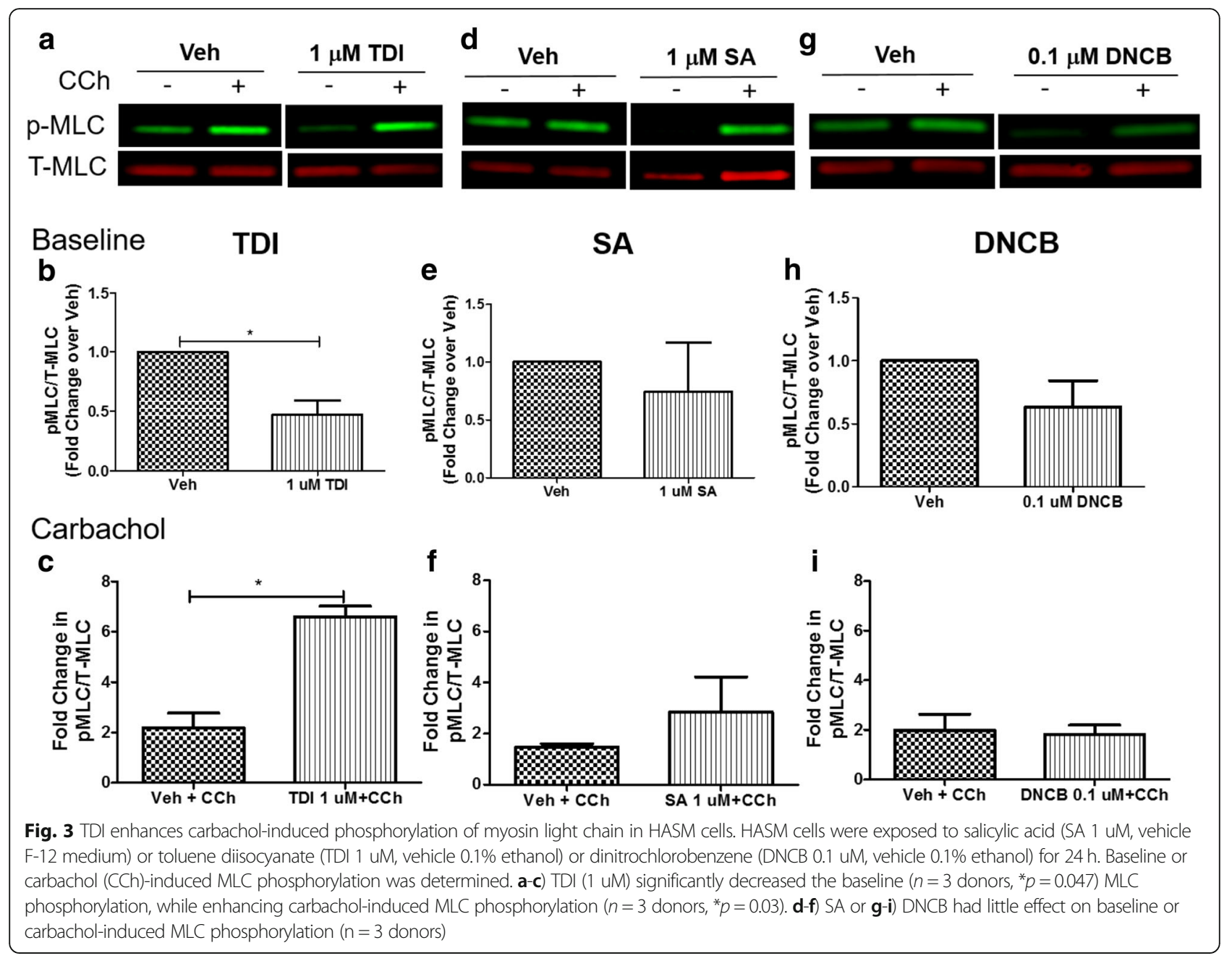

hPCLS, we tested whether DNCB induces oxidative injury in HASM cells. Nrf-2-mediated antioxidant response, as measured by NQO1 expression, tended to increase in the presence of DNCB (Fig. 5a \& d), although there was a higher level of inter-donor variability in this response ( $n=5$ donors). However, siRNA-mediated silencing of Nrf-2, abolished DNCB-induced NQO1 expression in HASM cells, suggesting DNCB induces this antioxidant system in HASM cells (Fig. 5b). TDI, on the other hand, had little effect on NQO1 expression in HASM cells (Fig. 5c \& e).

\section{Discussion}

The role of airway smooth muscle cells in AHR and asthma exacerbations is underestimated. We tested the hypothesis that three different toxicants, representing a non-sensitizing irritant (salicylic acid), respiratory sensitizer (toluene diisocyanate) and dermal sensitizer (dinitrochlorobenzene), modulate excitation-contraction coupling to induce AHR. Our findings show that the non-sensitizing irritant SA induces AHR in hPCLS, while the sensitizers TDI and DNCB had little effect. The toxicants had little effect on agonist-induced $\mathrm{Ca}^{2+}$ mobilization, while TDI potentiated carbachol-induced MLC phosphorylation in HASM cells. Our findings indicate that, (i) irritants such as salicylic acid may induce AHR independent of inflammatory mediator release, (ii) sensitizers, such as TDI, can act on airway smooth muscle cells to modulate EC coupling, and (iii) the dermal sensitizer DNCB induces Nrf-2 dependent antioxidant response in HASM cells without significant effects on EC coupling or AHR.

Irritant-induced asthma is generally associated with acute inhalation of noxious chemicals in occupational settings [36]. In some individuals, repeated exposure to inhaled irritants may lead to development of long term airway disorders such as reactive airway dysfunction syndrome, characterized by persistent AHR and airway inflammation [37]. SA is a ubiquitous chemical in cosmetic products, with a potential to be inhaled by consumers and cosmetic industry workers. Non-prescription cosmetic products contain $\sim 5 \%(w / v)$ of SA, which is equivalent to $362 \mathrm{mM}$ of active compound. Our findings 


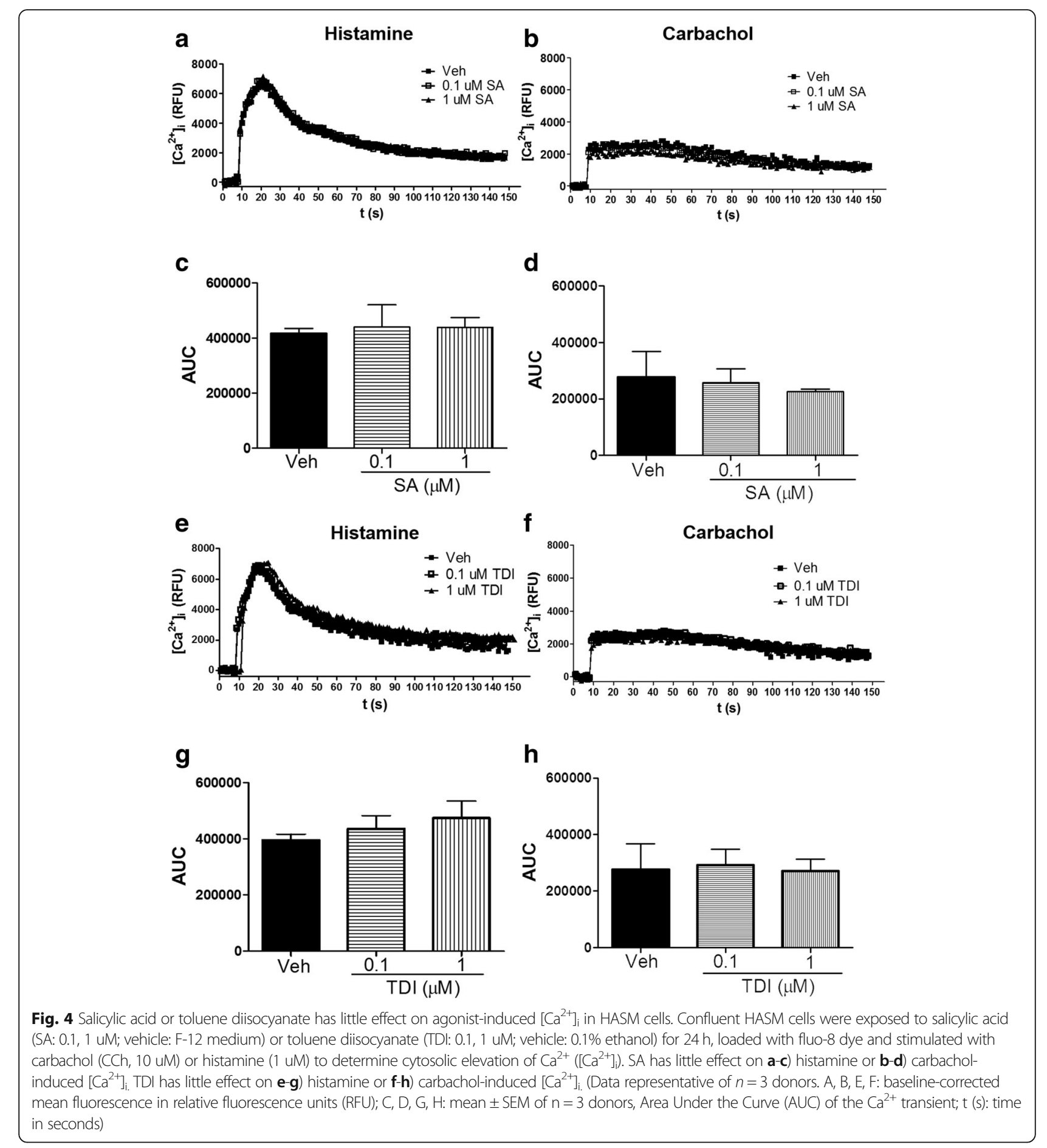

show that a concentration two orders of magnitude less than that of $5 \%(\mathrm{w} / \mathrm{v}) \mathrm{SA}$ is sufficient to enhance carbachol-induced bronchoconstriction in hPCLS, without inducing inflammatory mediator release. Well known respiratory irritants, such as chlorine, typically induce airway epithelial injury, inflammatory mediator release and oxidative stress [38].
Enhanced $\mathrm{Ca}^{2+}$ mobilization by contractile agonists is one of the mechanisms through which cytokines and certain toxicants mediate airway hyper-reactivity [23, 39]. Our past studies showed that toxicants could also amplify $\mathrm{Ca}^{2+}$ sensitization pathways in HASM cells to elicit AHR [1]. While SA induced AHR in PCLS with little effect on MLC phosphorylation, TDI amplified MLC 


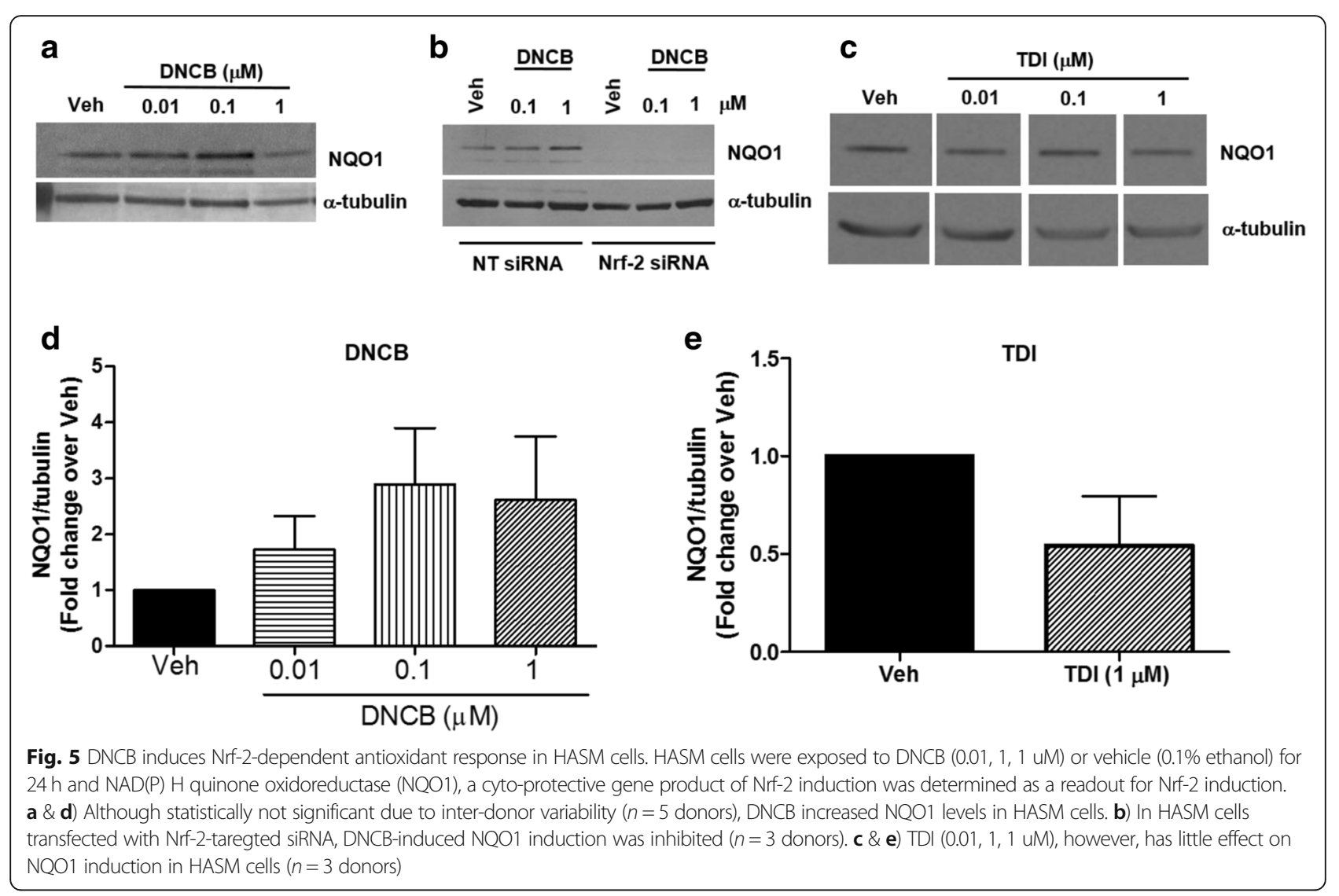

phosphorylation in HASM cells without enhancing bronchoconstriction. These observations suggest that SA-induced AHR potentially originates from actin polymerization in HASM cells [25]. Alternatively, airway epithelial cells may have a prominent role in SA-induced AHR. Airway epithelium provides the first line of physical barrier against inhaled toxicant and in airway diseases like asthma, injured airway epithelium allows these environmental toxicants to reach sub-epithelial tissues [40]. It is likely that airway epithelial cells primarily mediate SA-induced AHR in hPCLS, through paracrine modulation of ASM cells. Future studies will test this hypothesis using in vitro co-culture of HASM and air-liquid interface (ALI)-differentiated epithelial cells.

TDI exposure levels in occupational settings range between 5 and $20 \mathrm{ppb}$ [5]. Bronchial challenge experiments in human subjects found that TDI, at levels as low as 11 ppb, elicit asthmatic response in about $40 \%$ of the subjects [41]. Although the highest concentration of TDI $(1 \mu \mathrm{M})$ in our experiments translate to $\sim 170 \mathrm{ppb}$, this concentration was non-toxic and failed to elicit AHR in hPCLS. The lack of AHR by TDI, despite amplified MLC phosphorylation in HASM cells, supports the theory that SA mediates AHR through actin polymerization. Alternatively, it is plausible that airway epithelial cells in hPCLS have an inhibitory role on TDI-induced airway hyperreactivity. Airway epithelial cells provide the first line of defense against toxicants through their synthetic and physical barrier functions. In hPCLS, the synthetic role of epithelial cells is more relevant than the barrier function. Although Nrf-2-dependent antioxidant response is not induced by TDI in HASM cells, airway epithelial cells may mount an anti-oxidant defense upon TDI exposure to modulate their secretory response to TDI. Studies show that thioredoxin-dependent antioxidant mechanisms are upregulated in airway epithelial cells in response to exposure to pathogens and toxicants to confer protection $[42,43]$, suggesting similar mechanisms may be at play in hPCLS exposed to TDI. In order to test this hypothesis, future studies should directly measure cell shortening in HASM cells co-cultured with ALI-differentiated epithelial cells and treated with TDI $[35,44]$.

Clinically relevant DNCB doses have been reported from dermal sensitization studies in humans. Dermal challenge studies showed that DNCB levels ranging between $0.8-3.6 \mu \mathrm{g} . \mathrm{cm}^{-2}(\sim 4-18 \mu \mathrm{M})$ were capable of eliciting a skin reaction depending on the sensitizing DNCB dose [45]. The lack of AHR or inflammatory mediator release by sensitizing toxicant DNCB in hPCLS confirms earlier findings that circulating innate and adaptive immune cells may be necessary to mediate the effects of this toxicant. Since they are low molecular 
weight (LMW) antigens, TDI and DNCB need to form adducts with other proteins to become immunologically reactive antigens ("haptenized antigens") [46, 47]. It is likely that TDI or DNCB form such adducts with proteins in airway structural cells, to directly modulate the functions of those proteins. Amplified agonist-induced MLC phosphorylation by TDI in HASM cells may be the result of this non-specific adduct formation.

We previously reported that Nrf-2-dependent cyto-protective response had little effect on EC coupling in HASM cells [1]. Nrf2-dependent antioxidant response to DNCB is potentially beneficial since it counters ROS-mediated injury. DNCB induces Nrf2-dependent cyto-protective response in a variety of cell types [48]. Studies showed that DNCB-induced Nrf2 activity attenuated apoptosis in dendritic cells by modulating BCL2 expression [49]. It is likely that Nrf2 induction plays a similar role in HASM cells.

These toxicants, particularly the SA, may also act through neurogenic mechanisms to induce AHR. Our in vitro model has limitations to study neurogenic mechanisms. Similarly, lack of circulating inflammatory and immune cells in hPCLS restricts our interpretations to mechanisms in the airway structural cells.

\section{Conclusion}

In summary, our findings show that the non-sensitizing toxicant salicylic acid can evoke AHR in hPCLS, manifested as a leftward shift in the concentration response curve to carbachol. This occurs in the absence of inflammatory mediator release. The sensitizers TDI and DNCB, while modulating HASM cell signaling related to EC coupling and oxidative injury, failed to induce AHR in hPCLS. We can conclude that the investigated toxicants modulate airway structural cells, with a potential to induce AHR in susceptible individuals with airway diseases, such as asthma and COPD.

\section{Additional files}

Additional file 1: Table S1. List of cytokines/chemokines screened by Luminex Multi-analyte array (DOCX 293 kb)

Additional file 2: Figure S1. DNCB has little effect on carbachol-induced bronchoconstriction in hPCLS. Precision-cut human lung slices (hPCLS) from normal human lung donors were exposed DNCB (0.1-10 uM, vehicle $0.1 \%$ ethanol) for $24 \mathrm{~h}$, followed by a carbachol (CCh) concentration-response for bronchoconstriction. A-D) DNCB has little significant effect on carbacholinduced bronchoconstriction in hPCLS ( $n=3-8$ donors) (PDF $41 \mathrm{~kb}$ )

\section{Abbreviations}

$\left[\mathrm{Ca}^{2+}\right]_{i}$ : intracellular $\mathrm{Ca}^{2+}$ level; AHR: airway hyperresponsiveness; ALI: AirLiquid Interface; ASM: airway smooth muscle; AUC: Area Under the Curve; BCA: Bicinchoninic Acid; CaMKIl: $\mathrm{Ca}^{2+}$-calmodulin kinase; CCh: Carbachol; COPD: Chronic Obstructive Pulmonary Disease; DNCB: 1-chloro-2,4dinitrobenzene; EC: Excitation-Contraction; Emax: maximal response; FBS: Fetal Bovine Serum; FEV 1 : Forced Expiratory Volume in $1 \mathrm{~s}$; HASM: Human Airway Smooth Muscle; hPCLS: human precision-cut lung slices; IL: Interleukin; LDH: Lactate Dehydrogenase; $\log \mathrm{EC}_{50}$ : concentration of half-maximal response; MLC: Myosin Light Chain; NQO1: NAD(P) H Quinone Oxidoreductase 1; Nrf-2: Nuclear factor erythroid 2(NFE2)-related factor 2; pMLC: phosphorylated MLC; RFU: Relative Fluorescence Units; ROS: Reactive oxygen species; SA: Salicylic acid; TDI: Toluene diisocyanate; TNF-a: Tumor Necrosis Factor - a

\section{Acknowledgements}

We thank Dr. Nikaeta Sadekar (RIFM, Woodcliff Lake, NJ) for her critical suggestions to improve the manuscript.

\section{Funding}

The study was supported through a collaboration with Research Institute for Fragrance Materials (RIFM), Woodcliff Lake, NJ.

\section{Availability of data and materials}

The data generated and used during the current study are available from the corresponding author on reasonable request.

\section{Authors' contributions}

JJ, RAP, DB: Conception, experimental design, interpretation of results and preparation of manuscript; JJ, NK, GC \& WJ: Execution of experiments, data collection and interpretation of results. All authors read and approved the final manuscript.

\section{Ethics approval and consent to participate}

The human lung tissue was commercially obtained from the National Disease Research Interchange (NDRI) and the International Institute for the Advancement of Medicine (IIAM), from anonymous donors according to the procedures approved by the Rutgers University Institutional Review Board. The human tissue therefore is exempt from requiring IRB approval. HASM Cell lines and precision cut lung slices were only derived from the tissue procured from NDRI and IIAM

\section{Consent for publication}

Not applicable.

\section{Competing interests}

Dr. Danielle Botelho is a staff member at RIFM. Dr. Reynold Panettieri, Jr. was a member of RIFM Expert Panel. All other authors declare no competing interests.

\section{Publisher's Note}

Springer Nature remains neutral with regard to jurisdictional claims in published maps and institutional affiliations.

\section{Author details}

${ }^{1}$ Rutgers Institute for Translational Medicine and Science (RITMS), Rutgers, The State University of New Jersey, Rm\# 4276, 89 French Street, New Brunswick NJ08901, USA. ${ }^{2}$ Research Institute for Fragrance Materials (RIFM), Woodcliff Lake, New Jersey, USA

Received: 28 January 2019 Accepted: 26 March 2019

Published online: 11 April 2019

References

1. Jude J, et al. Formaldehyde induces rho-associated kinase activity to evoke airway Hyperresponsiveness. Am J Respir Cell Mol Biol. 2016;55(4):542-53.

2. Tarlo SM. Irritant-induced asthma in the workplace. Curr Allergy Asthma Rep. 2014;14(1):406.

3. Vandenplas $\mathrm{O}$, et al. EAACl position paper: irritant-induced asthma. Allergy. 2014;69(9):1141-53.

4. Esser PR, Martin SF. Pathomechanisms of contact sensitization. Curr Allergy Asthma Rep. 2017;17(12):83.

5. Collins, J., et al., Incidence of occupational asthma and exposure to toluene Diisocyanate in the United States toluene Diisocyanate production industry. J Occup Environ Med, 2017. 59 Suppl 12: p. S22-S27.

6. Padoan $\mathrm{M}$, et al. Long-term follow-up of toluene diisocyanate-induced asthma. Eur Respir J. 2003;21(4):637-40.

7. Ott MG, Diller WF, Jolly AT. Respiratory effects of toluene diisocyanate in the workplace: a discussion of exposure-response relationships. Crit Rev Toxicol. 2003;33(1):1-59. 
8. Huang J, et al. Immunological effects of toluene diisocyanate exposure on painters. Arch Environ Contam Toxicol. 1991;21(4):607-11.

9. Peters JM, et al. Acute respiratory effects in workers exposed to low levels of toluene diisocyanate (TDI). Arch Environ Health. 1968;16(5):642-7.

10. Wegman $\mathrm{DH}$, et al. A dose-response relationship in TDI workers. J Occup Med. 1974;16(4):258-60.

11. Watanabe $\mathrm{H}$, et al. Contact hypersensitivity: the mechanism of immune responses and T cell balance. J Interf Cytokine Res. 2002;22(4):407-12.

12. Arts $J \mathrm{H}$, et al. The respiratory local lymph node assay as a tool to study respiratory sensitizers. Toxicol Sci. 2008;106(2):423-34.

13. De Jong $\mathbf{W H}$, et al. Contact and respiratory sensitizers can be identified by cytokine profiles following inhalation exposure. Toxicology. 2009;261(3):103-11.

14. Kuper CF, et al. The contact allergen dinitrochlorobenzene (DNCB) and respiratory allergy in the Th2-prone Brown Norway rat. Toxicology. 2008; 246(2-3):213-21.

15. van Triel JJ, et al. Allergic inflammation in the upper respiratory tract of the rat upon repeated inhalation exposure to the contact allergen dinitrochlorobenzene (DNCB). Toxicology. 2010;269(1):73-80.

16. Lazaar AL, Panettieri RA Jr. Airway smooth muscle as a regulator of immune responses and bronchomotor tone. Clin Chest Med. 2006;27(1):53-69 vi.

17. Damera G, Tliba O, Panettieri RA Jr. Airway smooth muscle as an immunomodulatory cell. Pulm Pharmacol Ther. 2009;22(5):353-9.

18. Berridge MJ. Smooth muscle cell calcium activation mechanisms. J Physiol. 2008;586(Pt 21):5047-61.

19. Tansey MG, et al. Ca (2+)-dependent phosphorylation of myosin light chain kinase decreases the $\mathrm{Ca} 2+$ sensitivity of light chain phosphorylation within smooth muscle cells. J Biol Chem. 1994;269(13):9912-20.

20. Billington CK, Penn RB. Signaling and regulation of $G$ protein-coupled receptors in airway smooth muscle. Respir Res. 2003;4:2.

21. Feng J, et al. Inhibitory phosphorylation site for rho-associated kinase on smooth muscle myosin phosphatase. J Biol Chem. 1999;274(52):37385-90.

22. Aghajanian A, et al. Direct activation of RhoA by reactive oxygen species requires a redox-sensitive motif. PLoS One. 2009;4(11):e8045.

23. Hyvelin JM, et al. Cellular mechanisms of acrolein-induced alteration in calcium signaling in airway smooth muscle. Toxicol Appl Pharmacol. 2000; 164(2):176-83.

24. Yoshida $M$, et al. Ozone exposure may enhance airway smooth muscle contraction by increasing ca (2+) refilling of sarcoplasmic reticulum in Guinea pig. Pulm Pharmacol Ther. 2002;15(2):111-9.

25. Zhang $W$, et al. A novel role for RhoA GTPase in the regulation of airway smooth muscle contraction. Can J Physiol Pharmacol. 2015;93(2):129-36.

26. Panettieri RA, et al. A human airway smooth muscle cell line that retains physiological responsiveness. Am J Phys. 1989;256(2 Pt 1):C329-35.

27. Cooper PR, Panettieri RA Jr. Steroids completely reverse albuterol-induced beta (2)-adrenergic receptor tolerance in human small airways. J Allergy Clin Immunol. 2008;122(4):734-40.

28. Cooper PR, et al. C-027 inhibits IgE-mediated passive sensitization bronchoconstriction and acts as a histamine and serotonin antagonist in human airways. Allergy Asthma Proc. 2011;32(5):359-65.

29. Lauenstein $L$, et al. Assessment of immunotoxicity induced by chemicals in human precision-cut lung slices (PCLS). Toxicol in Vitro. 2014;28(4):588-99.

30. Dhingra N, Gulati N, Guttman-Yassky E. Mechanisms of contact sensitization offer insights into the role of barrier defects vs. intrinsic immune abnormalities as drivers of atopic dermatitis. J Invest Dermatol. 2013;133(10):2311-4.

31. Deshpande DA, et al. Modulation of calcium signaling by interleukin-13 in human airway smooth muscle: role of CD38/cyclic adenosine diphosphate ribose pathway. Am J Respir Cell Mol Biol. 2004;31(1):36-42.

32. Guedes AG, et al. Role of CD38 in TNF-alpha-induced airway hyperresponsiveness. Am J Physiol Lung Cell Mol Physiol. 2008;294(2):L290-9.

33. Jude JA, et al. Differential induction of CD38 expression by TNF-\{alpha\} in asthmatic airway smooth muscle cells. Am J Physiol Lung Cell Mol Physiol. 2010;299(6):L879-90.

34. Mahn $\mathrm{K}$, et al. Ca (2+) homeostasis and structural and functional remodelling of airway smooth muscle in asthma. Thorax. 2010;65(6):547-52.

35. Orfanos $\mathrm{S}$, et al. Obesity increases airway smooth muscle responses to contractile agonists. Am J Physiol Lung Cell Mol Physiol. 2018.

36. Brooks SM, Bernstein IL. Irritant-induced airway disorders. Immunol Allergy Clin N Am. 2011;31(4):747-68 vi.

37. Labrecque M. Irritant-induced asthma. Curr Opin Allergy Clin Immunol. 2012;12(2):140-4.
38. Martin JG, et al. Chlorine-induced injury to the airways in mice. Am J Respir Crit Care Med. 2003;168(5):568-74.

39. Jude JA, et al. Calcium signaling in airway smooth muscle. Proc Am Thorac Soc. 2008;5(1):15-22

40. Holgate ST, et al. The role of the airway epithelium and its interaction with environmental factors in asthma pathogenesis. Proc Am Thorac Soc. 2009;6(8):655-9.

41. Moscato G, et al. Toluene diisocyanate-induced asthma: clinical findings and bronchial responsiveness studies in 113 exposed subjects with work-related respiratory symptoms. J Occup Med. 1991;33(6):720-5.

42. Fekkar A, et al. Secretome of human bronchial epithelial cells in response to the fungal pathogen Aspergillus fumigatus analyzed by differential in-gel electrophoresis. J Infect Dis. 2012;205(7):1163-72.

43. Lee YC, et al. TRX-ASK1-JNK signaling regulation of cell density-dependent cytotoxicity in cigarette smoke-exposed human bronchial epithelial cells. Am J Physiol Lung Cell Mol Physiol. 2008;294(5):L921-31.

44. Yoo EJ, et al. Galpha12 facilitates shortening in human airway smooth muscle by modulating phosphoinositide 3-kinase-mediated activation in a RhoA-dependent manner. Br J Pharmacol. 2017;174(23):4383-95.

45. Friedmann PS. The relationships between exposure dose and response in induction and elicitation of contact hypersensitivity in humans. $\mathrm{Br} J$ Dermatol. 2007:157(6):1093-102.

46. Aleksic M, et al. Mass spectrometric identification of covalent adducts of the skin allergen 2,4-dinitro-1-chlorobenzene and model skin proteins. Toxicol in Vitro. 2008;22(5):1169-76.

47. Sabbioni $\mathrm{G}$, et al. Determination of albumin adducts of 4,4'methylenediphenyl diisocyanate after specific inhalative challenge tests in workers. Toxicol Lett. 2016;260:46-51.

48. Ade N, et al. HMOX1 and NQO1 genes are upregulated in response to contact sensitizers in dendritic cells and THP-1 cell line: role of the Keap1/ Nrf2 pathway. Toxicol Sci. 2009;107(2):451-60.

49. El Ali Z, et al. Dendritic cells' death induced by contact sensitizers is controlled by Nrf2 and depends on glutathione levels. Toxicol Appl Pharmacol. 2017;322:41-50.

Ready to submit your research? Choose BMC and benefit from:

- fast, convenient online submission

- thorough peer review by experienced researchers in your field

- rapid publication on acceptance

- support for research data, including large and complex data types

- gold Open Access which fosters wider collaboration and increased citations

- maximum visibility for your research: over $100 \mathrm{M}$ website views per year

At $\mathrm{BMC}$, research is always in progress.

Learn more biomedcentral.com/submissions 